126 BOOK REVIEWS

\title{
Éducation, transmission, rénovation à la Renaissance
}

Textes réunis par Bruno Pinchard et Pierre Servet. Ouverture de Jacqueline de Romilly

Genève : Droz, 2006, 340 p.

Les questions intellectuelles et spirituelles que pose l'humanisme à la Renaissance ne sont pas près d'être épuisées comme en témoigne la présente enquête sur les formes de la transmission des savoirs telle qu'elle paraît dans les actes du colloque qui s'est tenu du 3 au 6 décembre 2003 au Centre Jean Prévost de l'Université Jean Moulin - Lyon 3. On ne saurait mieux faire le point, en effet, sur les principales recherches contemporaines pour dégager quelques moments-clés d'un rayonnement dans la société européenne qui bénéficia à la Renaissance de nouveaux moyens de communication et de persuasion. On en jugera par les diverses contributions des spécialistes réunis ici.

Si le rôle fondateur des grands textes antiques (Platon, Cicéron, Augustin) reste essentiel, la construction de l'idéal « renaissant » a pu se faire aussi a contrario, par exemple en prenant le contre-pied de la logique platonicienne (J.-F. Mattéi), en gardant ses distances par rapport au pessimisme augustinien (J.-J. Wunenburger sur Thomas More) ou en redéfinissant l'art de la mémoire du patrimoine classique pour en faire un « art de vivre » (J.-C. Margolin et J. Domansky sur Érasme). Un véritable esprit de rénovation voit le jour, que ce soit pour susciter une nouvelle forme de magistère (O. Millet sur Calvin) ou pour instaurer une nouvelle méthode de lecture qui rompe avec celle des glossateurs et incorpore les textes anciens dans la culture de l'époque (J. Céard sur Budé). L'harmonie musicale elle-même sert de justification aux innovations que l'on tente alors de diffuser (P. Otaola-Gonzales).

Certaines communications analysent le nouveau type de relation qui s'instaure entre l'auteur et son livre (P. Servet sur Montaigne), entre l'enseignant et ses élèves (D. Bertrand sur la pédagogie jésuite), entre le censeur et le souverain (Th. Berns sur Bodin). Les guerres de religion, « ombre fatale » de l'humanisme, peuvent aussi se comprendre par contraste avec les visions utopiques de l'époque (C.-G. Dubois) que traversent parfois des spectacles curieusement dérangeants (B. Pinchard sur Rabelais). Dans les savoirs hermétiques et cabalistiques se mêlent des éléments platoniciens qui créent un univers syncrétique également troublant (M.J. B. Allen sur Ficin, Illana Zinguer sur Léon L’hébreu, E. Nuzzo sur G. Bruno). La question très actuelle de l'émergence du sujet moderne est elle-même abordée à travers l'étude des discours qui permettent l'énonciation $\mathrm{du} \ll$ moi $\gg$ depuis la pensée autonomiste d'un Nicolas de Cues (F. Vengeon) jusqu'au « souci de soi » tel que le conçoit Montaigne (J.-L. Vieillard-Baron). On complétera ces analyses en consultant les 
actes récemment parus de deux autres colloques : L'Émergence du sujet, éd. O. Pot (Genève : Droz, 2005) et Nature et paysages : L'émergence d'une nouvelle subjectivité à la Renaissance, éd. D. de Courcelles (Actes des journées d'études organisées par l’École nationales des chartes, 26 mars 2004 et 15 avril 2005).

À côté de ces communications résolument eurocentristes, on aura le plaisir de découvrir un autre regard sur un autre monde et une autre époque : la Perse du $\mathrm{XII}^{\mathrm{e}}$ siècle, où s'affrontent des penseurs à l'esprit curieusement très proche de leurs successeurs florentins. Au théologien Ghazâlî (1058-1111) s'oppose le philosophe néo-platonicien Sohravardî (1155-1191) et l'on observe alors une véritable « rupture de paradigme » dans l'histoire de la pensée islamique (C. Fleury). Comme quoi la Renaissance n'est pas un phénomène entièrement occidental, même si les modalités pratiques de son développement sont très différentes en Orient. Sans doute faut-il procéder avec prudence et éviter des assimilations rapides entre civilisations difficilement comparables. Mais il est parfois utile aux spécialistes de sortir de l'isolement auquel les obligent les impératifs méthodologiques de leur discipline. À l'ère du village global, il n'est pas vain de souhaiter de plus grands partages entre spécialistes de l'histoire des civilisations.

On nous permettra d'émettre un seul regret pour finir. Il est dommage que l'éditeur n'ait pas mis à la disposition du lecteur un appareil scientifique adéquat. Une bibliographie et un index des noms et des notions auraient été d'un grand secours, élargissant l'utilité de cet ouvrage érudit et répondant ainsi au vœu exprimé dans $l^{\prime} \ll$ Ouverture » par Jacqueline de Romilly.

FRANÇOIS RIGOLOT, Princeton University

\section{Agrippa d'Aubigné, Les Tragiques}

Édition critique établie et annotée par Jean-Raymond Fanlo

Champion classiques, série «Littératures », Paris : H. Champion, 2006, 1122 p.

Les éditions Champion republient dans leur jeune collection « Champion classiques » l'édition des Tragiques donnée par Jean-Raymond Fanlo en 2003, à partir de son édition de 1995 dans la collection des Textes de la Renaissance dirigée par Claude Blum, collection dans laquelle il vient aussi de faire paraître les Écrits politiques d'Aubigné. Les mérites de cet immense travail ne sont plus à vanter : texte donné selon les principes les plus novateurs de l'édition moderne, rendant sensible dans les variantes, après une enquête scrupuleuse, la diversité et la complexité des manuscrits - la restitution, en particulier, de la ponctuation originale ouvre un 